\title{
Intraoperative heparin resistance, severe tricuspid valve regurgitation and acute right ventricular failure following large right atrial myxoma excision in a post corona virus disease -2019 patient - A case report
}

Rudra Mahapatra ${ }^{1}$, Satyajeet Misra ${ }^{1}$, Siddhartha Sathia ${ }^{1}$, Shradha Gupta $^{1}$, Anirudh Elayat ${ }^{1}$, and Ramachandra Barik ${ }^{2}$

${ }^{1}$ AIIMS Bhubaneswar

${ }^{2}$ All India Institute of Medical Sciences Bhubaneswar

November 23, 2020

\begin{abstract}
ABSTRACT INTRODUCTION Tricuspid valve regurgitation (TR) and severe right ventricular (RV) systolic dysfunction may appear acutely after right atrial (RA) myxoma excision. Covid-19 infection may have a role in production of Heparin resistance. METHODS A case of post covid-19 infected RA myxoma was operated by surgical excision develop severe TR and acute right ventricular systolic dysfunction postoperatively for which Tricuspid valve ring annuloplasty was performed and RV was supported on Cardiopulmonary bypass (CPB). RESULTS RV dysfunction did not improve leading to cardiac arrest and death. Conclusion * Excision of RA myxoma can unmask the severe TR and severe RV systolic dysfunction not responsive to TV ring annuloplasty. * COVID-19 infection might have a role in production of heparin resistance. KEY WORDS: Covid-19, tricuspid annuloplasty, intraoperative heparin resistance, RV Dysfunction, cardiopulmonary bypass.
\end{abstract}

Title Page

Title -

Intraoperative heparin resistance, severe tricuspid valve regurgitation and acute right ventricular failure following large right atrial myxoma excision in a post corona virus disease - 2019 patient - A case report

\section{Authors}

Dr Rudrapratap Mahapatra* (M.Ch), Dr Satyajeet Misra (MD), Dr Siddhartha Sathia (M.Ch), Dr Shradha Gupta( MS), Dr Anirudh Elayat (MD), Dr Ramachandra Barik (DNB). Dr. RudraPratap Mahapatra*: M.Ch, Assistant Professor, Dept of Cardiothoracic Surgery, AIIMS, Bhubaneswar, E-mail -ctvs_rudra@aiimsbhubaneswar.edu.in ,ORCID id - 0000-0002-6561-6960 Dr. Satyajeet Misra: MD, Additional Professor \& HOD, Dept of Department of Anaesthesiology and critical Care, AIIMS Bhubaneswar, E-mail anaes_satyajeet@aiimsbhubaneswar.edu.in, ORCID id - 0000-0001-8097-0338 Dr. Siddhartha Sathia: M.Ch, Associate Professor \& HOD-I/C, Dept of Cardiothoracic surgery, AIIMS, Bhubaneswar, E-mail - ctvs_siddhartha@aiimsbhubaneswar.edu.in ORCID id - 0000-0001-7929-6200 Dr. Shradha Gupta: MS, Senior Resident (M.Ch), Dept of Cardiothoracic Surgery, AIIMS, Bhubaneswar, E-mail -shradha005@gmail.com ORCID id 0000-0003-2885-7420 Dr.Anirudh Elayat :MD, Senior Resident (PDF-Cardiothoracic Anaesthesia), Dept of Anaesthesiology and critical Care, AIIMS Bhubaneswar, E-mail- anirudhunnie7741@gmail.com ORCID id 
- 0000-0002-4090-5504Dr. Rama Chandra Barik: DNB, Associate Professor, Department of Cardiology, AIIMS Bhubaneswar, E-mail -Cardioramachandra@gmail.com ORCID id - 0000-0003-1965-8454

Short Running Title: Severe right ventricular Failure

*Corresponding author: Dr RudraPratap Mahapatra *: MCh, Assistant Professor, Dept of Cardiothoracic and Vascular Surgery, AIIMS, Bhubaneswar, Sijua, Patrapada, Bhubaneswar PIN-751019 (Odisha) E-mail id - ctvs_rudra@aiimsbhubaneswar.edu.in Telephone / Mobile No - 91-9437372413 / 919437677005Declaration :

1. None of the Manuscript's content have been previously published.

2. Data Availability - AIIMS -Bhubaneswar.

3. Funding : NIL

4. Conflict of interest : No conflict of interest.

5. Institutional review board approval / waiver: Not Applicable

6. Patient consent statement : Taken

\section{Hosted file}

Manuscript .pdf available at https://authorea.com/users/378135/articles/494689-intraoperativeheparin-resistance-severe-tricuspid-valve-regurgitation-and-acute-right-ventricularfailure-following-large-right-atrial-myxoma-excision-in-a-post-corona-virus-disease2019-patient-a-case-report 